\title{
Stem growth and root yield of cassava in inland valley \\ INFLUENCE OF TOPOSEQUENCE POSITION ON STEM GROWTH, BRANCHING HABIT AND ROOT YIELD OF CASSAVA IN INLAND VALLEY ECOLOGY
}

\author{
MOHAMED T. LAHAI ${ }^{1 *}$ AND INDIRA J. EKANAYAKE ${ }^{2}$ \\ ${ }^{1}$ Department of Crop Science, Njala University, PMB, Freetown, Sierra Leone \\ ${ }^{2}$ International Institute of Tropical Agriculture (IITA), Ibadan, Nigeria, c/o L. W. Lambourn \& Co.,Carolyn \\ House, 6 Dingwall Road, Croydon CR93EE, England. \\ Corresponding author. Phone: +232 33 838551. E-mail: drmtlahai@yahoo.com.
}

\begin{abstract}
Cassava is grown in both upland and inland valley ecologies (IVS). Water table depths in IVS significantly influence the productivity of cassava. A trial was conducted to evaluate the effects of toposequence position in inland valley ecology on stem growth, branching habit and root yield of cassava using a $4 x 4$ Latin Square design. There were significant differences among cultivars, toposequence positions, sites and years for stem growth parameters and root yield. High yielding cultivars (TMS 91/02324 and TMS 91/02327) were short and late branching, had one branching level and big stems, while low yielding ones (TMS 4(2)1425 and landrace) were early branching and had up to three branching levels. Cultivars with low branching habit produced higher number of stems than the profuse branching ones. Stem number correlated positively with yield at shallow water table site, indicating its importance as yield determinant in less branching cultivars at shallow water table depth. The number of branching levels and height to second and third branching levels correlated negatively with yield at shallow water table, suggesting that yield reduction due to high number of branches was more severe at shallow than deep water table. Drought stress stimulated forking in less branching cultivars, but reduced branching in high branching ones. Shallow water table and drought stress reduced stem number, stem size, height to forking and plant height, but the reduction was lower for high than low yielding cultivars. Therefore, stem growth parameters were generally higher in the valley fringe with deeper table than in the other positions with shallow water table.
\end{abstract}

KEY WORDS: Root yield, water table, Stem growth, Branching habit, Cassava, Inland valley

\section{INTRODUCTION}

The upper reaches of river systems comprising valley bottoms which may be submerged for part of the year, their hydromorphic fringes, and continuous upland slopes known as inland valleys (Andriesse et al., 1994) are a land resource of high production potential (Ekanayake et al., 1994) and cover substantial area. Inland valleys are estimated to occupy about 130 million hectares of land in tropical Africa (Andriesse, 1986) and between 2252 million hectares in West Africa (Andriesse et al., 1994). Rice is grown in the inland valleys during the wet season and yields are generally much higher than in the upland (IITA, 1988). In the dry season there is usually enough residual soil moisture or shallow groundwater table for upland crops to grow (Raunet, 1985).

Cassava is a popular dry season crop, grown to utilize residual soil moisture in inland valleys in West and Central Africa (Ekanayake et al., 1994). Breeding programs for cassava however, target the upland, but the constraint to production may be different from those in the inland valleys (Carsky et al., 1993). Cassava in inland valley is subjected to excess soil moisture stress during early and late season and to drought stress during mid-season growth stages (Ekanayake et al., 1994) which may reduce root yield. This is because water table is above the soil surface at planting period; recedes deep below the soil surface during the peak of the dry season; and again rises above the soil surface at harvesting (Mohamoud, 1994). Varietal differences in the performance of cassava have been reported for the inland valleys. Lahai et al. (1999) reported higher root yield for cultivar $87 / 29$ than $80 / 40$, while Jalloh (1998) showed that cultivars $85 / 40$ and 86/1 gave higher yield than other cultivars in the inland valley. The difference in yield between cassava cultivars is an indication of genetic variability which can be exploited in breeding programs. 
The difference in stem growth habit is an important variability that affects cassava root yield as the crop has simultaneous development of top and storage root, which are competing sinks for available carbohydrate (Cock, 1984; Ekanayake et al., 1998). This results in a delicate balance between shoot and root growth for maximum yield (Ramanujam, 1985). The appropriate balance between top growth and root growth is determined by top growth potential, which is largely determined by branching pattern (Cock et al., 1979); furthermore, it has been reported that the cassava plant type with high yield potential would branch at about 6 months after planting (Tan and Cock, 1979). Similarly, Manrique (1990) showed that dry matter partitioning to storage roots had little seasonal variation, but allocation to branches was more sensitive to the environment; in other words differences in root yield between seasons were mainly due to differences in branching habit.

However, the effects of stem growth habit on root yield of cassava have largely been reported for the upland ecology with a longer crop season (12-18 months), and very few studies have been conducted in this direction in the inland valley ecology with a short crop season (5-6 months) in the dry period. In this study we have observed stem growth and branching habit and their relationships with root yield of cassava in inland valley ecology.

\section{MATERIALS AND METHODS}

Sites, design, plant establishment and groundwater table depth measurement

The trials were conducted in small inland valleys at the International Institute of Tropical Agriculture (IITA) research farm at Ibadan $\left(7^{0} 30^{\prime} \mathrm{N}\right.$ and $3^{\circ} 54^{\prime} \mathrm{E}, 243 \mathrm{~m}$ above sea level) and on a farmer's field at Alabata $\left(4^{\circ} 46^{\prime} \mathrm{N}\right.$ and $2^{\circ} 34^{\prime}$ E, $210 \mathrm{~m}$ above sea level) in the forest-savanna transition zone of southwestern Nigeria. Weather data were monitored at the IITA weather station at Ibadan for both sites since they are about $3 \mathrm{~km}$ apart. During the experimental period mean minimum temperature in $2001\left(19.8^{\circ} \mathrm{C}\right)$ was higher than in $2002\left(19.1{ }^{\circ} \mathrm{C}\right)$, but mean maximum temperature was lower in $2001\left(27.5^{\circ} \mathrm{C}\right)$ than in $2002\left(28.4^{\circ} \mathrm{C}\right)$. Rainfall $(\mathrm{mm})$ and mean relative humidity (\%) were higher in $2001(955.9 \mathrm{~mm} ; 68 \%)$ than in $2002(642.6 \mathrm{~mm} ; 63 \%)$, but evaporation (mm) and solar radiation $(\mathrm{MJ} / \mathrm{m} /$ day) were higher in $2002(962.8 \mathrm{~mm} ; 13.49 \mathrm{MJ} / \mathrm{m} /$ day $)$ than in $2001(891.1 \mathrm{~mm} ; 13.09$ $\mathrm{MJ} / \mathrm{m} /$ day). The valley bottom soils classified as Tropaquents (Moormann et. al., 1975) were loamy sand at both sites. At Alabata, the soil had $\mathrm{pH}\left(\mathrm{H}_{2} \mathrm{O}\right)$ of 3.91 , organic $\mathrm{C}$ of $0.99 \%, \mathrm{~N}$, of $0.11 \%$, P of $7.43 \mathrm{mg} / \mathrm{kg}$ and $\mathrm{K}$ of $0.18(\mathrm{cmol} / \mathrm{kg})$ in 2001 . In 2002 , the values were $4.60,0.46 \%, 0.13 \%, 6.80 \mathrm{mg} / \mathrm{kg}$ and $0.20 \mathrm{cmol} / \mathrm{kg}$ for $\mathrm{pH}$, organic C, N, P and K, respectively. The corresponding values at Ibadan were $4.96,1.56 \%, 0.14 \%, 3.53 \mathrm{mg} / \mathrm{kg}$ and $0.08 \mathrm{cmol} / \mathrm{kg}$ in 2001 and $5.50,0.51 \%, 0.13 \%, 2.65 \mathrm{mg} / \mathrm{kg}$ and $0.10 \mathrm{cmol} / \mathrm{kg}$ in 2002 .

Three positions were marked out along the toposequence and designated as valley fringe, valley intermediate and valley bottom at each site. Each toposequence position had 16 flat-top mounds arranged in 4 x 4 Latin Square design. Each mound ( $2 \mathrm{~m} \times 2 \mathrm{~m}$ and $0.6 \mathrm{~m}$ high, spaced $1 \mathrm{~m}$ apart) was a plot. Three $2.1 \mathrm{~m}-$ long PVC pipes with diameter of $0.05 \mathrm{~m}$ were buried to a depth of $1.5 \mathrm{~m}$ in furrows between mounds in each toposequence position to serve as modified piezometers to monitor water table depth on weekly basis. This was done by placing a graduated meter ruler inside the PVC pipe so that the base of the ruler just touched the ground water surface and the reading from the surface to the top of the exposed pipe noted. The height of the exposed pipe which was the same as the mound height $(0.6 \mathrm{~m})$ was deducted from the reading obtained to give the water table depth below the mound surface.

Six stem cuttings $(0.25 \mathrm{~m}$ long) from healthy mature plants were planted on each mound at a spacing of $1 \mathrm{~m}$ between rows and $0.75 \mathrm{~m}$ between plants. Three improved cultivars (TMS 4(2)1425, TMS 91/02324 and TMS 91/02327) and a landrace (Isunikankiyan) were used in the study. Planting was done at Ibadan and Alabata on 12 February and 12 March 2001 in the first season and on 18 and 24 December 2001 in the second season, respectively. Hand weeding was done as required but no fertilizer was applied. The first and second season trials were terminated on 15 August 2001 and 23 July 2002 at Ibadan and on 13 September 2001 and 28 July 2002 at Alabata, respectively. 


\section{Stem growth and root yield of cassava in inland valley}

\section{Data Collection}

\section{Stem growth and storage root yield measurements}

Four plants were randomly selected and tagged in each plot a month after planting for various plant growth parameters. Data collected on the stem of cassava include number, the thickness using a vernier calliper, plant height, measured with a ruler from ground level to the top of the canopy and height to first and subsequent branching levels from the ground level to the point of forking: At harvest of the number of branching levels on all plants were counted in each plot while the weights of the stem and storage roots of all plants in each plot consisting of a total of 24 plants per cultivar per toposequence were also recorded. Sub-sample of fresh weight, (250g) was dried in a ventilated oven at $70^{\circ} \mathrm{C}$ for $48 \mathrm{~h}$ to determine dry weight.

\section{Statistical analyses}

Statistical analyses were performed using statistical analysis system (SAS) for Microsoft Windows, Release 6.12. Mixed model procedure using the restricted maximum likelihood method for the estimation of the random variance components was used for the analyses of variances. Rows and columns were the random effects and cultivars, toposequence position, location and year the fixed effects. Standard errors were used to detect differences between treatment means. Relationships between stem growth parameters and dry root yield, groundwater table depth and weather variables were explored by correlation analysis using PROC CORR procedure in SAS (SAS Institute, 1997).

\section{RESULTS}

\section{Groundwater table depth}

At both sites in each year, groundwater table depth decreased as rainfall increased. Water table was deeper at both sites in 2002 with lower rainfall than in 2001 with higher rainfall. It was also deeper in the fringe than the valley bottom and valley intermediate positions at both sites and in both years (Figure 1). In both years water table was deeper at Alabata than Ibadan. The shallowest water table depth ranged from 0.15 to $0.40 \mathrm{~m}$ in 2001 and 0.35 to $0.45 \mathrm{~m}$ in 2002 at Ibadan; from 0.45 to $0.50 \mathrm{~m}$ in 2001 and 0.51 to $0.58 \mathrm{~m}$ in 2002 at Alabata below the $0.6 \mathrm{~m}$-high mound surface.

\section{Dry storage root yield}

There were significant differences among cultivars, toposequence positions, sites and years for root yield. Generally, the improved cultivars outyielded the landrace, but TMS 91/02324 and TMS 91/02327 had similar and significantly higher yields than TMS 4(2)1425 across toposequence positions at each site in each year. There were significant toposequence position $\mathrm{x}$ site, toposequence position $\mathrm{x}$ year, site $\mathrm{x}$ year and toposequence position $\mathrm{x}$ site $\mathrm{x}$ year interactions for dry storage root yield of cassava. These interactions occurred as a result of the fact that across cultivars in 2001, the highest yield was recorded in valley bottom and the lowest in valley fringe at Alabata, while in 2002 at Alabata and in both years at Ibadan the highest yield was produced in the fringe and the lowest in valley bottom. On average, yield was about 55\% higher in 2001 than 2002 and 46\% higher at Alabata than Ibadan (Table 1). 
(a) Feb-Sept 2001
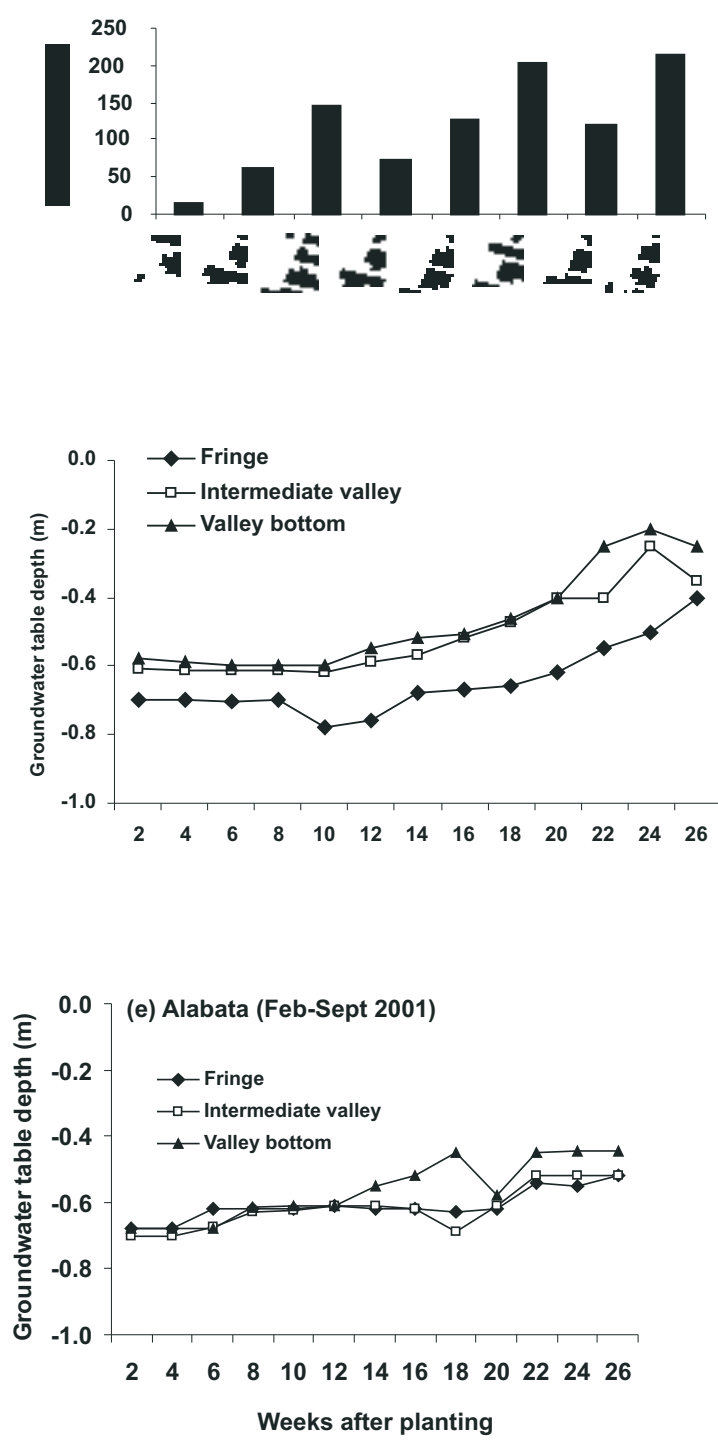

(b) Dec 2001-July 2002

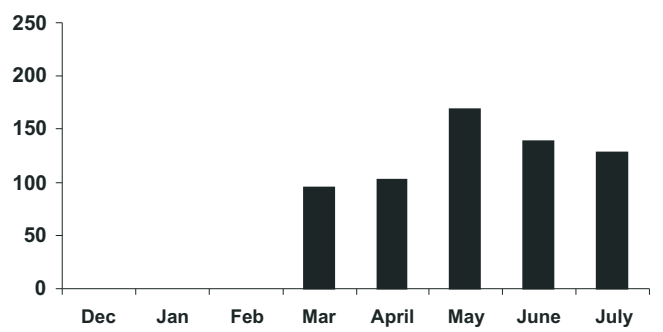

(d) Ibadan (Dec 2001-July 2002)
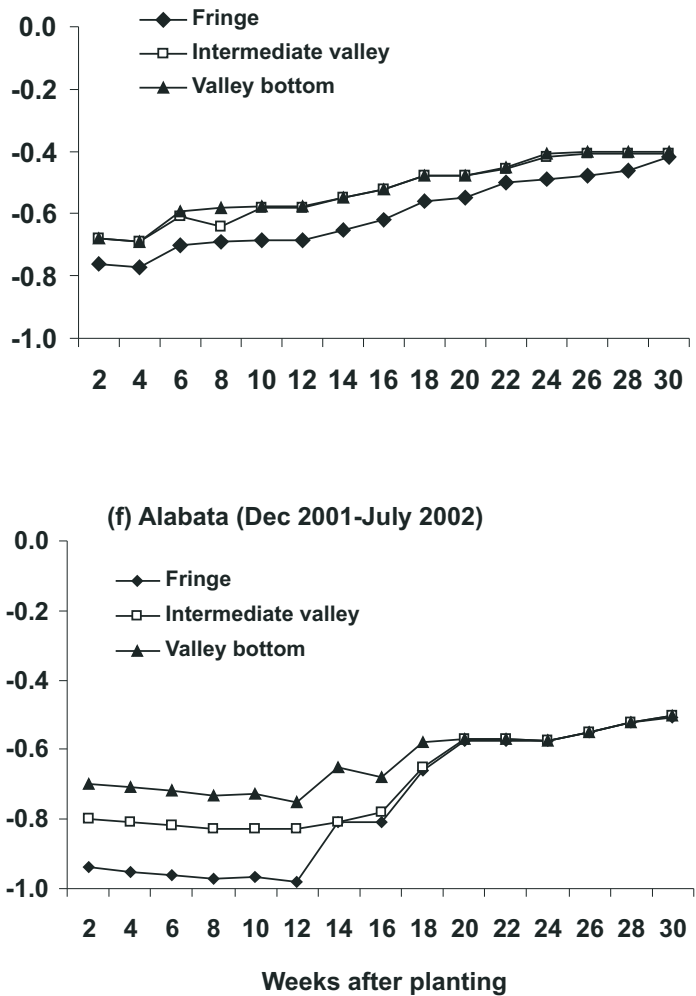

Figure 1. Variations in rainfall (at Ibadan) and in groundwater table depth during the 2001 and 2002 cropping seasons in inland valley at Ibadan and Alabata, Nigeria. 
Stem growth and root yield of cassava in inland valley

Table 1: Dry storage root yield ( $\left.\mathbf{h a}^{-1}\right)$ and number of stems per plant of four cassava cultivars in various toposequence positions at two inland valley sites in 2001-2002 in Nigeria

\begin{tabular}{|c|c|c|c|c|c|c|c|c|c|}
\hline \multirow[b]{3}{*}{ Site } & \multirow[b]{3}{*}{ Cultivar } & \multicolumn{4}{|l|}{2001} & \multicolumn{4}{|l|}{2002} \\
\hline & & \multicolumn{3}{|c|}{ Toposequence (Topo) } & \multirow[b]{2}{*}{$\begin{array}{l}\text { Cultivar } \\
\text { Mean }\end{array}$} & \multicolumn{3}{|c|}{ Toposequence (Topo) } & \multirow[b]{2}{*}{$\begin{array}{l}\text { Cultivar } \\
\text { mean }\end{array}$} \\
\hline & & Fringe & $\begin{array}{l}\text { Inter- } \\
\text { mediate }\end{array}$ & Bottom & & Fringe & $\begin{array}{l}\text { Inter- } \\
\text { mediate }\end{array}$ & Bottom & \\
\hline & & \multicolumn{8}{|c|}{ Dry storage root yield $\left(t h a^{-1}\right)$} \\
\hline \multirow[t]{8}{*}{ Alabata } & TMS4(2)1425 & 5.04 & 6.16 & 16.94 & 9.38 & 3.28 & 2.24 & 3.01 & 2.84 \\
\hline & TMS91/02324 & 4.68 & 4.71 & 17.24 & 8.88 & 4.12 & 4.01 & 4.66 & 4.26 \\
\hline & TMS91/02327 & 5.31 & 5.50 & 17.21 & 9.34 & 5.15 & 3.60 & 3.12 & 3.96 \\
\hline & Isunikankiyan & 4.17 & 4.98 & 15.23 & 8.13 & 1.86 & 1.97 & 0.95 & 1.59 \\
\hline & s. e \pm & 0.64 & 0.64 & 0.64 & 0.32 & 0.64 & 0.64 & 0.64 & 0.32 \\
\hline & Topo mean (s. e \pm 0.32 ) & 4.80 & 5.34 & 16.66 & & 3.60 & 2.96 & 2.94 & \\
\hline & Year mean $(\mathrm{s} . \mathrm{e} \pm 0.17)$ & 8.93 & & & & 3.17 & & & \\
\hline & Site mean (s. e \pm 0.13$)$ & 6.05 & & & & & & & \\
\hline \multirow[t]{9}{*}{ Ibadan } & TMS4(2)1425 & 4.56 & 4.22 & 3.23 & 4.00 & 3.18 & 2.30 & 1.67 & 2.38 \\
\hline & TMS91/02324 & 7.77 & 4.13 & 5.04 & 5.65 & 5.48 & 2.52 & 2.62 & 3.54 \\
\hline & TMS91/02327 & 6.12 & 4.51 & 4.41 & 5.01 & 5.14 & 2.38 & 2.04 & 3.19 \\
\hline & Isunikankiyan & 3.83 & 2.99 & 3.15 & 3.32 & 2.58 & 1.35 & 1.56 & 1.83 \\
\hline & s. e \pm & 0.64 & 0.64 & 0.64 & 0.32 & 0.64 & 0.64 & 0.64 & 0.32 \\
\hline & Topo mean (s. e \pm 0.32$)$ & 5.50 & 3.96 & 3.96 & & 4.10 & 2.14 & 1.97 & \\
\hline & Year mean $(\mathrm{s} . \mathrm{e} \pm 0.17)$ & 4.47 & & & & 2.74 & & & \\
\hline & Site mean $(\mathrm{s} . \mathrm{e} \pm 0.13)$ & 3.61 & & & & & & & \\
\hline & & \multicolumn{8}{|c|}{ Number of stems per plant } \\
\hline \multirow[t]{8}{*}{ Alabata } & TMS4(2)1425 & 2.5 & 2.3 & 2.0 & 2.3 & 1.5 & 1.6 & 1.4 & 1.5 \\
\hline & TMS91/02324 & 2.2 & 2.4 & 2.6 & 2.4 & 2.8 & 1.7 & 2.7 & 2.4 \\
\hline & TMS91/02327 & 2.4 & 2.5 & 2.3 & 2.4 & 2.6 & 2.1 & 2.4 & 2.4 \\
\hline & Isunikankiyan & 2.4 & 2.5 & 2.6 & 2.5 & 1.5 & 1.2 & 2.2 & 1.6 \\
\hline & s. e \pm & 0.05 & 0.05 & 0.05 & 0.06 & 0.05 & 0.05 & 0.05 & 0.06 \\
\hline & Topo mean (s. e \pm 0.05$)$ & 2.4 & 2.4 & 2.4 & & 2.1 & 1.7 & 2.2 & \\
\hline & Year mean $(\mathrm{s} . \mathrm{e} \pm 0.05)$ & 2.4 & & 2.4 & & 2.0 & & & \\
\hline & Site mean $(\mathrm{s} . \mathrm{e} \pm 0.05)$ & 2.2 & & & & & & & \\
\hline \multirow[t]{8}{*}{ Ibadan } & TMS4(2) 1425 & 2.1 & 2.1 & 2.2 & 2.1 & 1.9 & 1.7 & 1.9 & 1.8 \\
\hline & TMS91/02324 & 3.6 & 3.6 & 3.7 & 3.6 & 3.3 & 2.3 & 2.5 & 2.7 \\
\hline & TMS91/02327 & 3.2 & 2.5 & 2.7 & 2.8 & 3.2 & 2.5 & 2.7 & 2.8 \\
\hline & Isunikankiyan & 3.3 & 3.4 & 2.4 & 3.0 & 1.8 & 1.9 & 1.9 & 1.9 \\
\hline & s. e \pm & 0.05 & 0.05 & 0.05 & 0.06 & 0.05 & 0.05 & 0.05 & 0.06 \\
\hline & Topo mean (s. e \pm 0.05$)$ & 3.1 & 2.9 & 2.8 & & 2.6 & 2.1 & 2.3 & \\
\hline & Year mean $(\mathrm{s} . \mathrm{e} \pm 0.05)$ & 2.9 & & & & 2.3 & & & \\
\hline & Site mean $($ s. e \pm 0.05$)$ & 2.6 & & & & & & & \\
\hline
\end{tabular}

\section{Number of stems per plant}

Significant differences also existed among cultivars, toposequence positions, sites and years for number of stems produced. Generally, across toposequence positions at each site in each year, TMS 91/02324 produced significantly the highest number, followed by TMS 91/02327, while TMS 4(2)1425 had the lowest number of stems. Across cultivars, sites and years, plants in valley fringe generally had higher number of stems than those in the other positions. In 2001, plants had about $17 \%$ and $21 \%$ higher number of stems than in 2002 at Alabata and Ibadan, respectively. Plants also produced about $15 \%$ more stems at Ibadan than at Alabata. Interaction effects were not directional as cultivars changed their rankings only slightly over toposequence positions, sites and years (Table 1).

\section{Plant height}

Plant height differed significantly among cultivars, toposequence positions, sites and years. On average across toposequence positions, the landrace was significantly the tallest cultivar, followed by TMS 4(2)1425, while 
Mohamed T. Lahai and Indira J. Ekanayake

TMS 91/02324 and TMS 91/02327 attained identical heights, but were the shortest cultivars at both sites in both years. Across cultivars, plants in the fringe were taller than those in the other toposequence positions during both years at both sites. Plants were taller in 2001 than in 2002 by $49 \%$ at Alabata and $32 \%$ at Ibadan. Also plants at Alabata were about $46 \%$ taller than those at Ibadan. Toposequence position $\mathrm{x}$ site interaction was significant because at Alabata plants in valley intermediate were taller than those in valley bottom, but the former position had the shortest plants at Ibadan in both years (Table 2 ).

Table 2: Plant height $(\mathrm{m})$ and stem diameter $\times 10^{-1}(\mathrm{~m})$ of four cassava cultivars in various toposequence positions at two inland valley sites in 2001-2002 in Nigeria

\begin{tabular}{|c|c|c|c|c|c|c|c|c|c|}
\hline \multirow[b]{3}{*}{ Site } & \multirow[b]{3}{*}{ Cultivar } & \multicolumn{4}{|l|}{2001} & \multicolumn{4}{|l|}{2002} \\
\hline & & \multicolumn{3}{|c|}{ Toposequence (Topo) } & \multirow[b]{2}{*}{$\begin{array}{l}\text { Cultivar } \\
\text { Mean }\end{array}$} & \multicolumn{3}{|c|}{ Toposequence (Topo) } & \multirow[b]{2}{*}{$\begin{array}{l}\text { Cultivar } \\
\text { mean }\end{array}$} \\
\hline & & Fringe & $\begin{array}{l}\text { Inter- } \\
\text { mediate }\end{array}$ & Bottom & & Fringe & $\begin{array}{l}\text { Inter- } \\
\text { mediate }\end{array}$ & Bottom & \\
\hline & & \multicolumn{8}{|c|}{ Plant height } \\
\hline \multirow[t]{8}{*}{ Alabata } & TMS4(2)1425 & 3.77 & 2.77 & 2.65 & 3.06 & 1.86 & 1.20 & 1.45 & 1.50 \\
\hline & TMS91/02324 & 3.27 & 2.22 & 2.11 & 2.53 & 1.51 & 1.38 & 1.28 & 1.39 \\
\hline & TMS91/02327 & 2.85 & 2.22 & 1.95 & 2.34 & 1.68 & 1.28 & 1.15 & 1.37 \\
\hline & Isunikankiyan & 4.43 & 3.77 & 3.67 & 3.96 & 1.90 & 1.82 & 1.43 & 1.72 \\
\hline & s. e \pm & 0.03 & 0.03 & 0.03 & 0.03 & 0.03 & 0.03 & 0.03 & 0.03 \\
\hline & Topo mean $(\mathrm{s} . \mathrm{e} \pm 0.04)$ & 3.58 & 2.75 & 2.60 & & 1.74 & 1.42 & 1.33 & \\
\hline & Year mean (s. e \pm 0.02$)$ & 2.97 & & & & 1.50 & & & \\
\hline & Site mean $(\mathrm{s} . \mathrm{e} \pm 0.02)$ & 2.24 & & & & & & & \\
\hline \multirow[t]{9}{*}{ Ibadan } & TMS4(2)1425 & 1.79 & 1.33 & 1.30 & 1.47 & 1.02 & 0.66 & 0.73 & 0.80 \\
\hline & TMS91/02324 & 1.81 & 0.94 & 1.10 & 1.28 & 1.05 & 0.73 & 0.87 & 0.88 \\
\hline & TMS91/02327 & 1.63 & 0.96 & 1.07 & 1.22 & 1.13 & 0.70 & 0.83 & 0.89 \\
\hline & Isunikankiyan & 2.09 & 1.54 & 1.68 & 1.77 & 1.48 & 1.17 & 1.39 & 1.35 \\
\hline & s. e \pm & 0.03 & 0.03 & 0.03 & 0.03 & 0.03 & 0.03 & 0.03 & 0.03 \\
\hline & Topo mean (s. e \pm 0.04$)$ & 1.83 & 1.19 & 1.29 & & 1.17 & 0.82 & 0.96 & \\
\hline & Year mean (s. e \pm 0.02$)$ & 1.44 & & & & 0.98 & & & \\
\hline & Site mean (s. e \pm 0.02$)$ & 1.21 & & & & & & & \\
\hline & & \multicolumn{8}{|c|}{ Stem diameter } \\
\hline \multirow[t]{8}{*}{ Alabata } & TMS4(2)1425 & 0.27 & 0.24 & 0.24 & 0.25 & 0.19 & 0.15 & 0.18 & 0.17 \\
\hline & TMS91/02324 & 0.26 & 0.24 & 0.27 & 0.26 & 0.17 & 0.19 & 0.19 & 0.18 \\
\hline & TMS91/02327 & 0.26 & 0.24 & 0.23 & 0.24 & 0.16 & 0.17 & 0.16 & 0.16 \\
\hline & Isunikankiyan & 0.29 & 0.24 & 0.22 & 0.25 & 0.17 & 0.17 & 0.15 & 0.16 \\
\hline & s. e \pm & 0.002 & 0.002 & 0.002 & 0.003 & 0.002 & 0.002 & 0.002 & 0.003 \\
\hline & Topo mean (s.e \pm 0.003$)$ & 0.27 & 0.24 & 0.24 & & 0.17 & 0.17 & 0.17 & \\
\hline & Year mean $(\mathrm{s} . \mathrm{e} \pm 0.002)$ & 0.25 & & & & 0.17 & & & \\
\hline & Site mean (s. e \pm 0.002$)$ & 0.21 & & & & & & & \\
\hline \multirow[t]{8}{*}{ Ibadan } & TMS4(2)1425 & 0.27 & 0.21 & 0.19 & 0.22 & 0.22 & 0.15 & 0.15 & 0.17 \\
\hline & TMS91/02324 & 0.27 & 0.22 & 0.20 & 0.23 & 0.22 & 0.15 & 0.18 & 0.18 \\
\hline & TMS91/02327 & 0.24 & 0.22 & 0.20 & 0.22 & 0.20 & 0.14 & 0.16 & 0.17 \\
\hline & Isunikankiyan & 0.29 & 019 & 0.19 & 0.22 & 0.20 & 0.15 & 0.16 & 0.17 \\
\hline & s. e \pm & 0.002 & 0.002 & 0.002 & 0.003 & 0.002 & 0.002 & 0.002 & 0.003 \\
\hline & Topo mean (s.e \pm 0.003$)$ & 0.27 & 0.21 & 0.20 & & 0.21 & 0.15 & 0.16 & \\
\hline & Year mean (s. e \pm 0.002 ) & 0.23 & & & & 0.17 & & & \\
\hline & Site mean (s. e \pm 0.002$)$ & 0.20 & & & & & & & \\
\hline
\end{tabular}

\section{Stem diameter}

Stem size was also significantly affected by cultivar, toposequence position, site and year. The stems of TMS 91/02324 were bigger than those of TMS 91/02327 and the landrace, but apart from these, no other differences were significant across toposequence positions at each site in each year. Across cultivars, the biggest stems were produced in the valley fringe, while the other positions had similar and smaller stem sizes in both years at both sites. Stems were bigger by $32 \%$ at Alabata and by $26 \%$ at Ibadan in 2001 than in 2002 . Also stems were 


\section{Stem growth and root yield of cassava in inland valley}

bigger at Alabata than Ibadan. Site year interaction was significant. This was due to the fact that in 2001, plants at Alabata had bigger stems than those at Ibadan, but in 2002 the stem sizes were similar at both sites (Table 2).

\section{Number of branching levels}

There were distinct differences among cultivars and site for number of branching levels produced. In general, TMS 4(2)1425 produced the highest number, while TMS 91/02324 and TMS 91/02327 had similar and the lowest number of branches across toposequence positions at both sites and in both years. On average, plants at Ibadan had more branches than those at Alabata (Table 3). ANOVA exhibited several significant interaction effects. Cultivar x site interaction occurred because TMS 4(2)1425 and the landrace had higher number of branches at Ibadan than at Alabata, while TMS 91/02324 and TMS 91/02327 produced similar number of branches at both sites. There was cultivar x year interaction because TMS 4(2)1425 and the landrace had higher number of branches in 2001 than in 2002, but the reverse was noted for TMS 91/02324 and TMS 91/02327. Site $\mathrm{x}$ year interaction was also significant. At Alabata, similar number of branches was produced in both years, but at Ibadan higher number of branches were produced in 2002 than in 2001.

Table 3: Number of branching levels and mean height $(\mathrm{m})$ to first branching of four cassava cultivars in various toposequence positions at two inland valley sites in 2001-2002 in Nigeria

\begin{tabular}{|c|c|c|c|c|c|c|c|c|c|}
\hline \multirow[b]{3}{*}{ Site } & \multirow[b]{3}{*}{ Cultivar } & \multicolumn{4}{|l|}{2001} & \multicolumn{4}{|l|}{2002} \\
\hline & & \multicolumn{3}{|c|}{ Toposequence (Topo) } & \multirow[b]{2}{*}{$\begin{array}{l}\text { Cultivar } \\
\text { Mean }\end{array}$} & \multicolumn{3}{|c|}{ Toposequence (Topo) } & \multirow[b]{2}{*}{$\begin{array}{l}\text { Cultivar } \\
\text { mean }\end{array}$} \\
\hline & & Fringe & $\begin{array}{l}\text { Inter- } \\
\text { mediate }\end{array}$ & Bottom & & Fringe & $\begin{array}{l}\text { Inter- } \\
\text { mediate }\end{array}$ & Bottom & \\
\hline & & \multicolumn{8}{|c|}{ Number of branching levels } \\
\hline \multirow{8}{*}{ Alabata } & TMS4(2)1425 & 2.2 & 1.5 & 2.3 & 2.0 & 1.2 & 1.0 & 1.2 & 1.1 \\
\hline & TMS91/02324 & 0.7 & 0.3 & 0.8 & 0.6 & 1.7 & 1.4 & 1.3 & 1.5 \\
\hline & TMS91/02327 & 0.8 & 0.3 & 0.5 & 0.5 & 1.5 & 1.2 & 1.9 & 1.5 \\
\hline & Isunikankiyan & 0.9 & 2.5 & 2.0 & 1.8 & 0.7 & 1.3 & 1.0 & 1.0 \\
\hline & s. e \pm & 0.13 & 0.13 & 0.13 & 0.07 & 0.13 & 0.13 & 0.13 & 0.07 \\
\hline & Topo mean (s. e \pm 0.03 ) & 1.2 & 1.2 & 1.4 & & 1.3 & 1.2 & 1.4 & \\
\hline & Year mean (s. e \pm 0.04$)$ & 1.3 & & & & 1.3 & & & \\
\hline & Site mean $($ s. e \pm 0.02$)$ & 1.3 & & & & & & & \\
\hline \multirow[t]{9}{*}{ Ibadan } & TMS4(2) 1425 & 2.1 & 2.1 & 2.0 & 2.1 & 2.1 & 2.0 & 2.0 & 2.0 \\
\hline & TMS91/02324 & 1.0 & 1.0 & 0.9 & 1.0 & 1.7 & 1.5 & 1.3 & 1.5 \\
\hline & TMS91/02327 & 1.0 & 1.0 & 0.6 & 0.9 & 1.5 & 2.0 & 1.5 & 1.7 \\
\hline & Isunikankiyan & 1.8 & 1.4 & 1.6 & 1.6 & 1.9 & 2.0 & 2.0 & 2.0 \\
\hline & s. e \pm & 0.13 & 0.13 & 0.13 & 0.07 & 0.13 & 0.13 & 0.13 & 0.07 \\
\hline & Topo mean (s. e \pm 0.03 ) & 1.5 & 1.4 & 1.3 & & 1.8 & 1.9 & 1.7 & \\
\hline & Year mean $(\mathrm{s} . \mathrm{e} \pm 0.04)$ & 1.4 & & & & 1.8 & & & \\
\hline & Site mean $(\mathrm{s} . \mathrm{e} \pm 0.02)$ & 1.6 & & & & & & & \\
\hline & & \multicolumn{8}{|c|}{ Height to first branching } \\
\hline \multirow{8}{*}{ Alabata } & TMS4(2)1425 & 1.07 & 1.14 & 0.93 & 1.05 & 0.49 & 0.40 & 0.57 & 0.49 \\
\hline & TMS91/02324 & 1.77 & 1.04 & 1.12 & 1.31 & 0.71 & 0.72 & 0.88 & 0.77 \\
\hline & TMS91/02327 & 1.27 & 1.40 & 0.95 & 1.21 & 0.77 & 0.64 & 0.65 & 0.69 \\
\hline & Isunikankiyan & 2.03 & 1.77 & 1.23 & 1.68 & 0.65 & 1.23 & 0.69 & 0.85 \\
\hline & s. e \pm & 0.13 & 0.13 & 0.13 & 0.07 & 0.13 & 0.13 & 0.13 & 0.07 \\
\hline & Topo mean (s. e \pm 0.07$)$ & 1.54 & 1.34 & 1.06 & & 0.65 & 0.75 & 0.70 & \\
\hline & Year mean $(\mathrm{s} . \mathrm{e} \pm 0.04)$ & 1.31 & & & & 0.70 & & & \\
\hline & Site mean (s. e \pm 0.03$)$ & 1.01 & & & & & & & \\
\hline \multirow[t]{8}{*}{ Ibadan } & TMS4(2) 1425 & 0.89 & 0.50 & 0.48 & 0.62 & 0.39 & 0.33 & 0.34 & 0.35 \\
\hline & TMS91/02324 & 0.91 & 0.56 & 0.66 & 0.71 & 0.68 & 0.72 & 0.49 & 0.63 \\
\hline & TMS91/02327 & 0.82 & 0.50 & 0.75 & 0.69 & 0.50 & 0.70 & 0.62 & 0.61 \\
\hline & Isunikankiyan & 1.16 & 0.85 & 1.13 & 1.03 & 1.05 & 0.96 & 0.94 & 0.98 \\
\hline & s. e \pm & 0.13 & 0.13 & 0.13 & 0.07 & 0.13 & 0.13 & 0.13 & 0.07 \\
\hline & Topo mean (s. e \pm 0.07$)$ & 0.95 & 0.60 & 0.76 & & 0.66 & 0.68 & 0.60 & \\
\hline & Year mean $(\mathrm{s} . \mathrm{e} \pm 0.04)$ & 0.77 & & & & 0.65 & & & \\
\hline & Site mean (s. e \pm 0.03 ) & 0.71 & & & & & & & \\
\hline
\end{tabular}

Height to branching levels

Height to branching levels varied significantly among cultivars, toposequence position, sites and years. Generally, height to first branching was lower for TMS 4(2)1425 than the other cultivars. The landrace was the 
tallest at all branching levels. No significant differences were observed between TMS 91/02324 and TMS 91/02327, both of which did not branch beyond the first branching level across toposequence positions in each year at both sites. Across cultivars, heights to the three branching levels were significantly higher for plants in the fringe than the other positions at both sites in both years (Tables 3 and 4). In general, plants at Alabata were taller at first branching than at Ibadan. At both sites, plants were taller at first branching in 2001 than in 2002 (Table 3). Heights to second and third branching levels were higher at Alabata than Ibadan (Table 4). Toposequence position x year interaction was significant for height to first branching. In 2001, the fringe had the tallest plants, but in 2002, valley intermediate produced the tallest plants at first branching. Interaction effects for height to second and third branching were not directional as cultivars changed their rankings only slightly over toposequence position, site and year.

Table 4: Mean heights $(\mathrm{m})$ to second and third branching of four cassava cultivars in various toposequence positions at two inland valley sites in 2001-2002 in Nigeria

\begin{tabular}{|c|c|c|c|c|c|c|c|c|c|}
\hline \multirow[b]{3}{*}{ Site } & \multirow[b]{3}{*}{ Cultivar } & \multicolumn{4}{|l|}{2001} & \multicolumn{4}{|l|}{2002} \\
\hline & & \multicolumn{3}{|c|}{ Toposequence (Topo) } & \multirow[b]{2}{*}{$\begin{array}{l}\text { Cultivar } \\
\text { Mean }\end{array}$} & \multicolumn{3}{|c|}{ Toposequence (Topo) } & \multirow[b]{2}{*}{$\begin{array}{l}\text { Cultivar } \\
\text { mean }\end{array}$} \\
\hline & & Fringe & $\begin{array}{l}\text { Inter- } \\
\text { mediate }\end{array}$ & Bottom & & Fringe & $\begin{array}{l}\text { Inter- } \\
\text { mediate }\end{array}$ & Bottom & \\
\hline & & \multicolumn{8}{|c|}{ Height to second branching } \\
\hline \multirow{8}{*}{ Alabata } & TMS4(2)1425 & 1.95 & 1.45 & 1.83 & 1.74 & 1.39 & 0.89 & 1.27 & 1.18 \\
\hline & TMS91/02324 & 0.00 & 0.00 & 0.00 & $0.00 *$ & 0.00 & 0.00 & 0.00 & $0.00 *$ \\
\hline & TMS91/02327 & 0.00 & 0.00 & 0.00 & $0.00^{*}$ & 0.00 & 0.00 & 0.00 & $0.00 *$ \\
\hline & Isunikankiyan & 2.74 & 2.17 & 1.98 & 2.30 & 1.91 & 1.34 & 1.15 & 1.47 \\
\hline & s. e \pm & 0.12 & 0.12 & 0.12 & 0.07 & 0.12 & 0.12 & 0.12 & 0.07 \\
\hline & Topo mean $(\mathrm{s} . \mathrm{e} \pm 0.07)$ & 2.35 & 1.81 & 1.91 & & 1.65 & 1.12 & 1.21 & \\
\hline & Year mean (s. e \pm 0.04$)$ & 2.02 & & & & 1.33 & & & \\
\hline & Site mean $(\mathrm{s} . \mathrm{e} \pm 0.03)$ & 1.68 & & & & & & & \\
\hline \multirow[t]{9}{*}{ Ibadan } & TMS4(2)1425 & 1.36 & 0.92 & 0.92 & 1.07 & 1.09 & 0.65 & 0.62 & 0.79 \\
\hline & TMS91/02324 & 0.00 & 0.00 & 0.00 & $0.00^{*}$ & 0.00 & 0.00 & 0.00 & $0.00 *$ \\
\hline & TMS91/02327 & 0.00 & 0.00 & 0.00 & $0.00 *$ & 0.00 & 0.00 & 0.00 & $0.00 *$ \\
\hline & Isunikankiyan & 1.69 & 1.28 & 1.51 & 1.49 & 1.60 & 1.23 & 1.46 & 1.43 \\
\hline & s. e \pm & 0.12 & 0.12 & 0.12 & 0.07 & 0.12 & 0.12 & 0.12 & 0.07 \\
\hline & Topo mean (s. e \pm 0.07$)$ & 1.53 & 1.10 & 1.22 & & 1.35 & 0.94 & 1.04 & \\
\hline & Year mean (s. e \pm 0.04$)$ & 1.28 & & & & 1.11 & & & \\
\hline & Site mean (s. e \pm 0.03 ) & 1.20 & & & & & & & \\
\hline & & \multicolumn{8}{|c|}{ Height to third branching } \\
\hline \multirow{8}{*}{ Alabata } & TMS4(2)1425 & 2.80 & 2.68 & 2.63 & 2.70 & 2.24 & 2.12 & 2.07 & 2.14 \\
\hline & TMS91/02324 & 0.00 & 0.00 & 0.00 & $0.00 *$ & 0.00 & 0.00 & 0.00 & $0.00 *$ \\
\hline & TMS91/02327 & 0.00 & 0.00 & 0.00 & $0.00 *$ & 0.00 & 0.00 & 0.00 & $0.00 *$ \\
\hline & Isunikankiyan & 3.32 & 3.33 & 3.05 & 3.23 & 2.49 & 2.50 & 2.22 & 2.40 \\
\hline & s. e \pm & 0.08 & 0.08 & 0.08 & 0.07 & 0.08 & 0.08 & 0.08 & 0.07 \\
\hline & Topo mean (s. e \pm 0.07 ) & 3.06 & 3.01 & 2.84 & & 2.37 & 2.31 & 2.15 & \\
\hline & Year mean $(\mathrm{s} . \mathrm{e} \pm 0.04)$ & 2.97 & & & & 2.28 & & & \\
\hline & Site mean $(\mathrm{s} . \mathrm{e} \pm 0.03)$ & 2.63 & & & & & & & \\
\hline \multirow[t]{8}{*}{ Ibadan } & TMS4(2)1425 & 1.65 & 1.19 & 1.08 & 1.31 & 1.38 & 0.93 & 0.81 & 1.04 \\
\hline & TMS91/02324 & 0.00 & 0.00 & 0.00 & $0.00 *$ & 0.00 & 0.00 & 0.00 & $0.00 *$ \\
\hline & TMS91/02327 & 0.00 & 0.00 & 0.00 & $0.00^{*}$ & 0.00 & 0.00 & 0.00 & $0.00 *$ \\
\hline & Isunikankiyan & 1.97 & 1.97 & 1.96 & 1.97 & 1.92 & 1.90 & 1.87 & 1.90 \\
\hline & s. e \pm & 0.08 & 0.08 & 0.08 & 0.07 & 0.08 & 0.08 & 0.08 & 0.07 \\
\hline & Topo mean (s. e \pm 0.07$)$ & 1.81 & 1.58 & 1.52 & & 1.65 & 1.42 & 1.34 & \\
\hline & Year mean $($ s. e \pm 0.04$)$ & 1.64 & & & & 1.47 & & & \\
\hline & Site mean (s. e \pm 0.03$)$ & 1.56 & & & & & & & \\
\hline
\end{tabular}

* Did not produce second and third branching levels

Niger Agric. J. 40 No. 1 (2009): 132 - 144 


\section{Stem growth and root yield of cassava in inland valley}

\section{Association of stem growth parameters with yield, water table depth and weather}

Stem diameter correlated positively with root yield at both sites and with number of stems and plant height at Ibadan (Table 5). The number of branching levels and height to second and third branching correlated negatively with yield at Ibadan. Water table depth and evaporation rate, solar radiation and maximum temperature related negatively with stem diameter, stem number and plant height and height at first branching at Ibadan. In contrast, plant height, height at first branching, stem number and diameter correlated positively with rainfall, minimum temperature and relative humidity, (Table 5).

Table 5: Correlations between selected leaf and stem characters of cassava and tuberous root yield $(n=32)$, groundwater table depth $(n=32)$ and weather variables $(n=64)$ in inland valley

\begin{tabular}{|c|c|c|c|c|c|c|c|c|c|c|c|c|c|c|c|c|c|}
\hline \multirow[b]{2}{*}{ Stems characters } & \multicolumn{2}{|c|}{ Storage root yield $\left(\mathrm{t} \mathrm{ha}^{-1}\right)$} & \multicolumn{4}{|c|}{ Groundwater table depth $(\mathrm{m})$} & \multicolumn{11}{|c|}{ Weather variable } \\
\hline & $P$ & $\mathrm{R}$ & $\mathrm{r}$ & $\mathrm{p}$ & $\mathrm{r}$ & $\mathrm{p}$ & $\mathrm{R}$ & $\mathrm{p}$ & $\mathrm{R}$ & $\mathrm{p}$ & $\mathrm{r}$ & $\mathrm{p}$ & $\mathrm{r}$ & $\mathrm{p}$ & $\mathrm{r}$ & $\mathrm{p}$ & $\mathrm{p}$ \\
\hline Number of stems & 0.320 .0786 & 0.650 .0001 & -0.18 & 0.3234 & -0.36 & 0.0401 & 0.43 & 0.0004 & -0.43 & 0.0004 & -0.43 & 0.0004 & 0.43 & 0.0004 & -0.43 & 0.0004 & $0.43 \quad 0.0004$ \\
\hline Plant height & 0.330 .0638 & 0.380 .0296 & 0.24 & 0.1900 & -0.57 & 0.0007 & 0.54 & 0.0001 & -0.54 & 0.0001 & -0.54 & 0.0001 & 0.54 & 0.0001 & -0.54 & 0.0001 & 0.540 .0001 \\
\hline No. of branching levels & 0.090 .6151 & -0.570 .0007 & -0.10 & 0.5863 & 0.19 & 0.3104 & -0.21 & 0.0949 & 0.21 & 0.0949 & 0.21 & 0.0949 & -0.21 & 0.0949 & 0.21 & 0.0949 & -0.210 .0949 \\
\hline Height to $1^{\text {st }}$ branching & $0.22 \quad 0.2258$ & 0.070 .6862 & 0.19 & 0.2926 & -0.33 & 0.0690 & 0.49 & 0.0001 & -0.49 & 0.0001 & -0.49 & 0.0001 & 0.49 & 0.0001 & -0.49 & 0.0001 & 0.490 .0001 \\
\hline Height to $2^{\text {nd }}$ branching & -0.100 .5683 & -0.430 .0134 & 0.10 & 0.5903 & -0.08 & 0.6748 & 0.02 & 0.8785 & -0.02 & 0.8785 & -0.02 & 0.8785 & 0.02 & 0.8785 & -0.02 & 0.8785 & 0.020 .8785 \\
\hline Height to $3^{\text {rd }}$ branching & -0.130 .4861 & -0.440 .0113 & 0.05 & 0.7742 & -0.06 & 0.7429 & 0.01 & 0.9579 & -0.01 & 0.9579 & -0.01 & 0.9579 & 0.01 & 0.9579 & -0.01 & 0.9579 & $0.01 \quad 0.9579$ \\
\hline
\end{tabular}

\section{DISCUSSION}

TMS 91/02324 had the highest number of stems, followed by TMS 91/02327, while TMS 4(2)1425 had the lowest stem number. TMS 91/02324 and TMS 91/02327 compensated for low branching habit by producing more number of stems. Number of stems correlated positively with root yield at both sites, but the correlation was better at Ibadan than Alabata, indicating that stem number may be an important determinant of yield in inland valley, particularly at shallow water table. High stem number increased leaf area index, which may have contributed to the high root yields of TMS 91/02324 and TMS 91/02327. TMS 4(2)1425 produced few stems largely because of its inherent branching habit. Number of stems produced correlated negatively with water table depth at the shallow water table site at Ibadan, but at Alabata with deep water table, the correlation was not significant. This suggests that shallow water table depth reduces number of stems probably due to stress caused by water-logging conditions at shallow water table depth. This was supported by the fact that at Ibadan, number of stems in valley fringe with deep water table was higher than in the other positions with shallow water table. Shallow water table depth causes waterlogging which impedes root respiration and results in stunted growth of cassava (Mohamoud, 1994). Number of stems in 2001 was higher than in 2002 by $17 \%$ at Alabata and by $21 \%$ at Ibadan. This was partly due to the dry, hot and less humid weather conditions that prevailed in 2002 compared to 2001. Dry periods (Irikura et al., 1979; Keating, 1981) and temperatures above $27^{\circ} \mathrm{C}$ (Manrique, 1990) reduce stem number. In this study, the mean maximum temperature in 2002 was $28^{\circ} \mathrm{C}$ with a high evaporation rate and no rainfall during the first three months of growth likely reduced stem production. However, in 2002, TMS 91/02324 and TMS 91/02327 produced higher number of stems than the other cultivars, indicating their high level of tolerance to adverse weather conditions in inland valley.

The results revealed that the two shortest improved cultivars (TMS 91/02324 and TMS 91/02327) had the highest root yield while the landrace the tallest cultivar recorded the lowest yield in all toposequence positions. 


\section{Mohamed T. Lahai and Indira J. Ekanayake}

This corroborates findings of Lahai et al. (1994) who reported that the low yielding ability of local cultivars appeared to be partly associated with production of less leaf area due to tall stems with few branches. The high yield in the short cultivars may be due to their high harvest index, as they partitioned less dry matter to the stems than the tall cultivar. However, plant height correlated positively with root yield, but the correlation was only significant at Ibadan, the shallow water table site. Okogbenin et al. (1999) reported that although plant height is genetically controlled, the full realisation of the genetic potential depends on the environment. At the shallow water table site, all the cultivars were taller in valley fringe with deep water table where root yield was higher than valley bottom with shallow water table and hence the positive correlation between plant height and root yield at this site. The non significant correlation between plant height and yield at Alabata was partly due the production of very tall plants in valley fringe, which gave lower root yields than the short plants in valley bottom in 2001. This likely caused more dry matter accumulation in the stems at the expense of allocation to storage roots, resulting in lower yield in valley fringe than valley bottom. The results agreed with Lahai et al. (1994) who reported lower yield in plants with tall stems than those with short stems. Water table depth correlated negatively with plant height at the shallow water table site, indicating that shallow water table depth reduces plant height. This was supported by the fact that plants at Alabata site and in the valley fringe at both sites with deep water table were significantly taller than those at Ibadan site and valley bottom with shallow water table. The results are in line with the findings of Mohamoud (1994) who noted symptoms of chlorosis and stunted growth on cassava grown on low mounds $(30-60 \mathrm{~cm})$ at a shallow water table site, while no such symptoms were present at the deep water table site. Plants were taller in 2001 than in 2002 by $49 \%$ at Alabata and $32 \%$ at Ibadan. As is the case with root yield, plant height correlated positively with rainfall, minimum temperature and relative humidity, but negatively with evaporation rate, solar radiation and maximum temperature. In 2001, rainfall was relatively well distributed, with low temperature and high relative humidity, whereas 2002 had low and erratic rainfall, with no rains for the first three months, coupled with high solar radiation resulting in high evaporation and temperature and low relative humidity. Plants were thus more stressed in 2002, culminating in shorter plants and lower yield than in 2001 with more favourable weather conditions.

Stem diameter correlated positively with root yield at both sites, suggesting that the bigger the stem the higher the root yield. The high yield of TMS 91/02324 may therefore partly be attributed to its bigger stems. Most of the dry matter produced by cassava is allocated to the stems, leaves and tuberous roots (Kasele et al., 1998; Lahai et al., 1999). The proportions of dry matter allocated to the leaves and fibrous roots decrease with time, while the proportions partitioned to storage roots and stems increase with time (George et al., 1998; Kasele et al., 1998; Lahai et al., 1999). This suggests that growth of stem and storage root is a direct reflection of crop growth rate. Bigger stems usually have higher amount of nutrients and are therefore able to support higher LAI and root yield. Molina and El-Sharkawy (1995) showed that big stems with higher nutrient content produced higher LAI than small stems with low nutrient content. There was a marked negative correlation between stem diameter and water table depth at the shallow water table site, but correlation at the deep water table site was not significant (Table 5), suggesting that shallow water table depth reduces stem size. Consequently, stems at Alabata site and in the fringe at both sites with deep water table were bigger than at Ibadan site and valley bottom with shallow water table. Stem size was bigger in 2001 than in 2002 at both sites, which may be related to more favourable weather conditions in the first than second season.

The growth pattern differed among the cultivars with TMS 91/02324 and TMS 91/02327 having zero to one branching level, while the landrace and TMS 4(2)1425 had from one to three branching levels. However, the number of plants with three branching levels was higher for TMS 4(2)1425 than for the landrace. To compensate for the low branching habit, TMS 91/02324 and TMS 91/02327 produced high stem numbers and with the multiple shoot system and low plant height, their canopy structures were more compact and therefore intercepted light more efficiently, which may have contributed to their high yields. Shoot apices and storage roots compete for available carbohydrate (Enyi, 1972; Tan and Cock, 1979). The low number of branches in TMS 91/02324 and TMS 91/02327 likely reduced the competition between shoot apices and storage roots and

Niger Agric. J. 40 No. 1 (2009): 132 - 144

$-141-$ 


\section{Stem growth and root yield of cassava in inland valley}

more carbohydrate was available for root bulking resulting in high yields, while TMS 4(2)1425 and the landrace with high number of branches increased this competition with less assimilates for root bulking, which contributed to their low yields. There was marked negative correlation between number of branches as well as heights at second and third branching and root yield at the shallow water table site, but the correlation at the deep water table site was not significant. This indicated that yield reduction due to high number of branches, particularly second and third branching was more severe at shallow than deep water table, likely due to reduction in yield due to high number of branches and reduction in yield due to shallow water table. As with plant height, height to branching levels was higher in the fringe and also at Alabata with deep water table, suggesting that shallow water table reduced height to forking.

Forking started at 2 months after planting (MAP) in TMS 4(2)1425, whereas in TMS 91/02324 and TMS 91/02327 even at 6 MAP, only a few plants had forked. This was supported by height to first, second and third branching levels which were much lower for TMS 4(2)1425 than the other cultivars, signifying early branching for TMS 4(2)1425 and late forking for TMS 91/02324 and TMS 91/02327 with the highest yields. This was partly because in late branching cultivars, shoot apices competed less with storage roots for assimilates compared with early branching cultivars. The results agreed with Tan and Cock (1979) who showed that late branching at about 6 MAP leads to high yield.

As is the case with plant height, height at first branching correlated positively with rainfall, minimum temperature and relative humidity, but negatively with evaporation, solar radiation and maximum temperature and at both sites plants were taller at first branching in 2001 than in 2002. This may be related to favourable weather conditions in 2001 than in 2002. However, number of forks in the cultivars differed between years. TMS 4(2)1425 and the landrace with higher number of branches generally had more branches in 2001 with high rainfall than in 2002 with low rainfall, whereas TMS 91/02324 and TMS 91/02327 with lower number of branches had more forking levels in 2002 than in 2001. Connor and Cock (1981) noted that the interval between forking is increased by water stress, which is consistent with TMS 4(2)1425 and the landrace, but at variance with TMS 91/02324 and TMS 91/02327. Results suggest that wet weather increased forking in TMS 4(2)1425 and the landrace, whereas dry weather stimulated forking in TMS 91/02324 and TMS 91/02327. These results partly agreed with Indira and Ramanujam (1984) who reported that dry matter partitioning to tops decreased in leafy cultivars (TMS 4(2)1425), but increased in less leafy cultivars (TMS 91/02324 and TMS 91/02327) during drought period.

\section{CONCLUSION}

There were significant differences among cultivars, toposequence positions, sites and years for stem growth parameters and root yield. Cultivars with low branching habit produced higher number of stems than the profuse branching ones. High stem number, which increases LAI, may be an important determinant of yield in less branching cultivars. High yielding cultivars were short and late branching and had one branching level and big stems. Yield reduction due to high number of branches, particularly second and third branching levels was more severe at shallow than deep water table. Shallow water table and drought stress reduced stem number, stem size, height to forking and plant height. Drought stress stimulated forking in less branching cultivars, but reduced branching in high branching ones in inland valleys.

\section{ACKNOWLEDGEMENTS}

We thank IITA for financing this study and the staff of cassava physiology unit at IITA for assisting with field operations. 


\section{REFERENCES}

Andriesse W. (1986). Area and distribution. In: Juo A.S.R., Lowe J.A. (eds.). The wetlands and rice in subSaharan Africa. Proc. of International Conference held at Ibadan, Nigeria. Ibadan: IITA, pp 15-30.

Andriesse W, Fresco L.O., Van Duivenbooden N., Windmeizer P.N. (1994). Multi-scale characterization of Inland Valley agro-ecosystems in West Africa. Netherlands Journal of Agricultural Science 42: 159-179.

Baker G.R., Fukai S., Wilson G.L. (1989). The response of cassava to water deficits at various stages of growth in the subtropics. Australian Journal of Agricultural Research 40: 517-528.

Carsky R.J., Walker P., Hauser S., Dashiell, K., Dixon A.G.O. (1993). Response of selected crop associations to ground water table depth in an Inland Valley. Field Crops Research 34: 1-13.

Cock J.H. (1984). Cassava. In: Goldsworthy PR, Fisher NM (eds.) The Physiology of Tropical Field Crops, John Wiley and Sons, New York, pp 529-549.

Cock J.H., Franklin D., Sandoval G., Juri P. (1979). The ideal cassava plant for maximum yield. Crop Science 19:271-279.

Connor D.J., Cock J.H., Parra G.E. (1981). The response of cassava to water shortage. I Growth and yield. Field Crops Research 4: 181-200.

Ekanayake I.J., Dixon A.G.O., Asiedu R., Izac A-M.N. (1994). Improved cassava for Inland valley agroecosystems. In: Akoroda M.O. (ed.) Root Crop for Food Security in Africa: Proceedings of fifth Triennial Symposium of the International Society for Tropical Root Crops- Africa Branch held at Kampala, Uganda. Ibadan: IITA/ International Society for Tropical Root Crops, pp 204-208.

Ekanayake I.J., Okarter U.C., Adeleke M.T.V. (1998). Dry season dust alters photosynthetic characteristics of field grown cassava in the dry season in two agroecozones of Nigeria. In: Akoroda M.O., Ekanayake I.J. (eds.) Root Crops and Poverty Alleviation, Proceedings of Sixth Symposium of ISTRC-AB held at Lilongwe, Malawi, Ibadan: IITA/ International Society for Tropical Root Crops, p. 402.

Enyi B.A.C. (1972). The effects of spacing on growth, development and yield of single and multi-shoot plants of cassava (Manihot esculenta Crantz). II. Physiological factors. East African Agricultural and Forestry Journal 38: 27-34.

George J.B., Lahai M.T., Ekanayake I.J., Dahniya M.T. (1998). Growth rates and yield of three cassava cultivars on the Njala gravelly upland soils in Sierra Leone. In: Akoroda M.O., Ekanayake I.J. (eds) Root Crops and Poverty Alleviation, Proceedings of Sixth Symposium of ISTRC-AB held at Lilongwe, Malawi, Ibadan: IITA/ International Society for Tropical Root Crops, pp. 382-387.

IITA(1988). Annual Report for 1987. Ibadan, Nigeria: International Institute of Tropical Agriculture.

Indira P., Ramanujam T. (1984). Proline accumulation, relative water content and dry matter in cassava under moisture stress. Indian Journal of Agricultural Sciences 54: 336-337.

Irikura Y., Cock J.H., Kawano K. (1979). The physiological basis of genotype-temperature interactions in cassava. Field Crops Research 2: 227-239.

Niger Agric. J. 40 No. 1 (2009): 132 - 144

$-143-$ 


\section{Stem growth and root yield of cassava in inland valley}

Jalloh A. (1998). Productivity of cassava in the inland valley swamp in Sierra Leone. Final technical report submitted to the inland valley consortium co-ordinator, West African Rice Development Association (WARDA) Bouake, Ivory Coast. 42pp.

Kasele I.N., Ekanayake I.J., Dixon A.G.O. (1998). Preliminary investigations on photosynthetic capacity and nutrient uptake of polyploid cassava (Manihot esculenta Crantz). In: Akoroda M.O., Ekanayake I.J. (eds) Root Crops and Poverty Alleviation, Proceedings of Sixth Symposium of ISTRC-AB held at Lilongwe, Malawi, Ibadan: IITA/ International Society for Tropical Root Crops, p. 403.

Keating B.A. (1981). Environmental effects on growth and development of cassava (Manihot esculenta Crantz) with special reference to photoperiod and temperature. $\mathrm{PhD}$ thesis, Department of Agriculture, University of Queensland, Australia.

Lahai M.T., George J.B., Ekanayake I.J. (1999). Cassava (Manihot esculenta Crantz) growth indices, root yield and its components in upland and inland valley ecologies of Sierra Leone. Journal of Agronomy and Crop Science 182: 239-247.

Lahai M.T., Gbani A.B., Dahniya M.T., Tucker M.J., George J.B., James P., John J.I., Winnebah R.T.A., Banya G.S., Tarawalli A.R., Williams C.O., Jalloh V. (1994). Cassava variety and fertiliser on-farm trials in Sierra Leone. In: Akoroda M.O. (ed.) Root Crop for Food Security in Africa: Proceedings of fifth Triennial Symposium of the International Society for Tropical Root Crops- Africa Branch held at Kampala, Uganda. Ibadan: IITA/ International Society for Tropical Root Crops, pp. 176-180.

Manrique L.A. (1990). Plant morphology of cassava during summer and winter. Agronomy Journal 82: 881886.

Mohamoud Y.M. (1994). Effect of mound height and cassava cultivar on cassava performance under a fluctuating water table. Agricultural Water Management 26: 201-211.

Molina J.L., El-Sharkawy M.A. (1995). Increasing crop productivity in cassava by fertilising production of planting material. Field Crops Research 44:151-157.

Moormann, F.R., Lal R., Juo A.S.R. (1975). The soils of IITA. IITA Tech. Bull. No.3, Ibadan, Nigeria.

Okogbenin E., Ekanayake I.J., Porto M.C.M. (1999). Effect of planting materials and soil moisture on cassava performance in the semi-arid Sudan Savannah belt of Nigeria. African Crop Science Journal 1:21-33.

Ramanujam T. (1985). Leaf density profile and efficiency in partitioning dry matter among high and low yielding cultivars of cassava (Manihot esculenta Crantz). Field Crops Research 10: 291-303.

Raunet M. (1985). Bas-fonds et riziculture en Afrique- Approche structurale comparative. L'Agronomie Tropicale 40: 181-201.

SAS Institute (1997). SAS/STAT User's Guide, Version 6, Fourth Edition. Cary, NC, USA: SAS Institute.

Tan S.L., Cock J.H. (1979). Branching habit as a yield determinant in cassava. Field Crops Research 2: 281289. 